\title{
Top quark pair production and modeling via QCD in CMS
}

\author{
Juan R. González Fernández on behalf of the CMS Collaboration* \\ Universidad de Oviedo \\ E-mail: juan.rodrigo.gonzalez.fernandezecern.ch
}

\begin{abstract}
Measurements of the inclusive and differential top quark pair (t $\bar{t}$ ) production cross section at centre-of-mass energies of $13 \mathrm{TeV}$ and $5.02 \mathrm{TeV}$ are presented, performed using CMS data collected in 2015 and 2016. The inclusive cross section is measured in the lepton+jets, dilepton and fully hadronic channels. Top quark pair differential cross sections are measured and are given as functions of various kinematic observables of (anti)top quark, the tt system, and of the jets and leptons in the final state. Furthermore, the multiplicity and kinematic distributions of the additional jets produced in $\mathrm{t} \overline{\mathrm{t}}$ events are also investigated and its modeling is compared for several generators. A new tune of parameters is developed for some of the generators. In addition, first measurements of top quark pair production with additional $b$ quarks in the final state are presented. Furthermore, searches for four top quark production in CMS are also presented.
\end{abstract}

XXV International Workshop on Deep-Inelastic Scattering and Related Subjects

3-7 April 2017

University of Birmingham, $U K$

\footnotetext{
*Speaker.
} 


\section{Introduction}

The top quark is the heaviest particle known and the only quark that decays before hadronizing, and thus gives direct access to its properties. With its large mass, it plays a crucial role in electroweak loop corrections, providing indirect constraints on the mass of the Higgs boson. Top quark measurements also provide important input to QCD predictions. Moreover, various scenarios of physics beyond the standard model (SM) expect the top quark to couple to new particles.

Top quarks are produced at the LHC mostly in pairs ( $\mathrm{t} \overline{\mathrm{t}})$ via the strong interaction. The top quark decays almost exclusively into a $\mathrm{W}$ boson and a $\mathrm{b}$ quark, therefore, the decay of the $\mathrm{W}$ bosons define the $t \bar{t}$ final states: two leptons, two neutrinos and two $b$ jets (dilepton channel), one lepton, one neutrino and four jets, out of which two arise from a b quark (lepton+jets channel) or with six jets, out of which two stem from a $b$ quark (all hadronic channel). In this document, I present a review of the most recent results of tit production cross sections (inclusive and differential), $\mathrm{t} \overline{\mathrm{t}}$ production in association with $\mathrm{b}$ quarks ( $\mathrm{t} \overline{\mathrm{t}} \mathrm{b}$ ) and search for four top quark ( $\mathrm{t} \mathrm{t} t \overline{\mathrm{t}}$ ) production in different final states. The measurements are performed by the CMS Collaboration [1] using data from the LHC at centre-of-mass energies from 5.02 to $13 \mathrm{TeV}$.

\section{Inclusive $t \bar{t}$ cross sections}

The latest measurements of $\mathrm{t} \overline{\mathrm{t}}$ inclusive cross sections at $13 \mathrm{TeV}$ with up to $2.3 \mathrm{fb}^{-1}$ of data are presented. Also, a recent measurement of țt production cross section in proton-proton collisions at a centre-of-mass energy of $5.02 \mathrm{TeV}$ is shown.

\subsection{Inclusive cross sections at $\sqrt{s}=13 \mathrm{TeV}$}

Different measurements of the t⿱t production cross section have been made by CMS in different final states (dilepton, lepton + jets and full hadronic) using first data at $13 \mathrm{TeV}$, collected in 2015 . All of them are in agreement with the NNLO+NNLL SM prediction [3], which has a value of $\sigma_{t \bar{t}}^{t h}=832_{-19}^{+20}($ scales $) \pm 35\left(\mathrm{PDF}+\alpha_{s}\right) \mathrm{pb}$.

\subsubsection{Dilepton channel}

A recent measurement in the dilepton channel using $2.2 \mathrm{fb}^{-1}$ of data [5] supersedes the first measurement at this centre-of-mass energy of the $t \bar{t}$ production cross section by CMS [4]. This measurement is performed using events with an electron-muon $(e \mu)$ pair with opposite sign and invariant mass of at least $20 \mathrm{GeV}$. At least two reconstructed jets of $30 \mathrm{GeV}$ and $|\eta| \leq 2.4$ are required, and the presence of at least one b-tagged jet (b-jet). Most background contributions from other SM processes, almost negligible with the exceptoion of single-top production, are estimated from Monte Carlo (MC). Contribution from Drell-Yan (DY) events and nonprompt background are estimated using data-driven (DD) techniques. Main systematic uncertainties come from t⿱亠t modeling and jet energy scale.

In Figure 1 the jet multiplicity is shown after the dilepton selection.

The measured cross section in the dilepton channel is $\sigma_{t \bar{t}}=815 \pm 9$ (stat) \pm 38 (syst) \pm 19 (lumi) $\mathrm{pb}$, with a total relative precision of $5.3 \%$. 


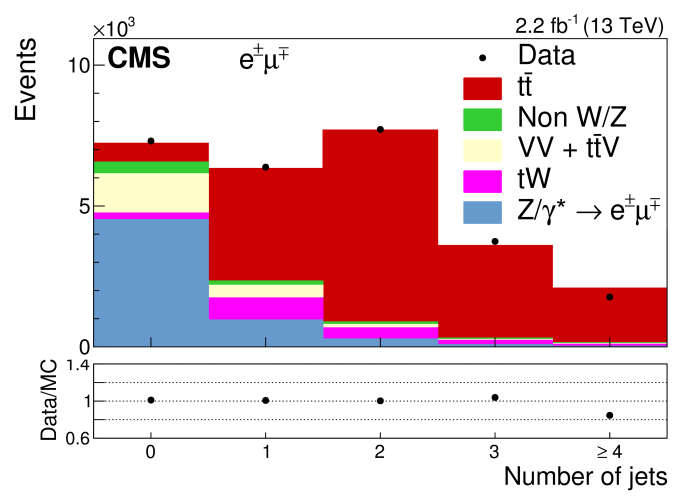

Figure 1: Distribution of the jet multiplicity for events with one $e^{ \pm} \mu^{\mp}$ pair [5].

\subsubsection{One lepton + jets}

A very precise measurement of the $t \bar{t}$ cross section was recently published by CMS in the lepton+jets channel [6]. Events with at least one electron or one muon are selected and further are categorized by jet and b-jet multiplicities. Main backgrounds are W+jets and QCD multijet, which are estimated from data in the low-jet and low-b-jet categories. The cross section is extracted by performing a simultaneous binned likelihood fit to all the categories, where systematic uncertainties are taken into account as nuisance parameters and are constrained. The largest uncertainties are the $\mathrm{W}+\mathrm{jets}$ background estimation, the uncertainty on the luminosity and on the $t \bar{t}$ modeling.

In Figure 2 the numbers of events in each category are shown.

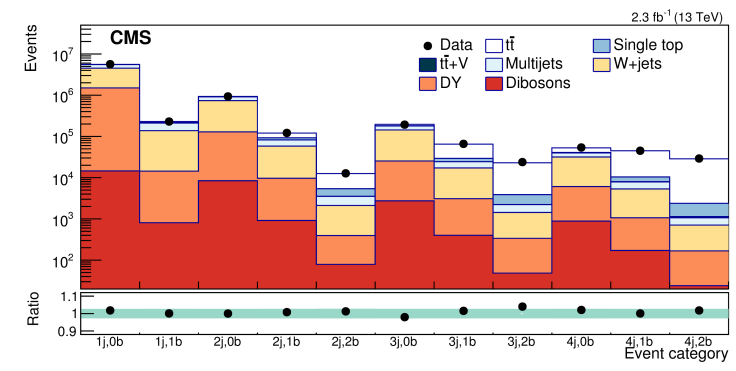

Figure 2: Yields for data and expected signal and background events for each of the categories of jet (j) and b-tagged jet (b) multiplicity in events with one electron or muon [6].

The measured cross section in this channel is $\sigma_{t \bar{t}}=834.6 \pm 2.5$ (stat) \pm 22.8 (syst) \pm 22.5 (lumi) $\mathrm{pb}$, with a total relative precision of $3.85 \%$.

\subsubsection{All hadronic channel}

A recent measurement of the $t \bar{t}$ cross section was also done in the full hadronic channel, including a study of the boosted regime [7]. Although the large branching fraction in this channel, the estimation of the huge QCD background introduces larger uncertainties. Top mass $\left(m_{t}\right)$ is reconstructed and an unbinned likelihood maximum fit is used to extract the signal strength.

In Figure 3 the $m_{t}$ distribution is shown. 


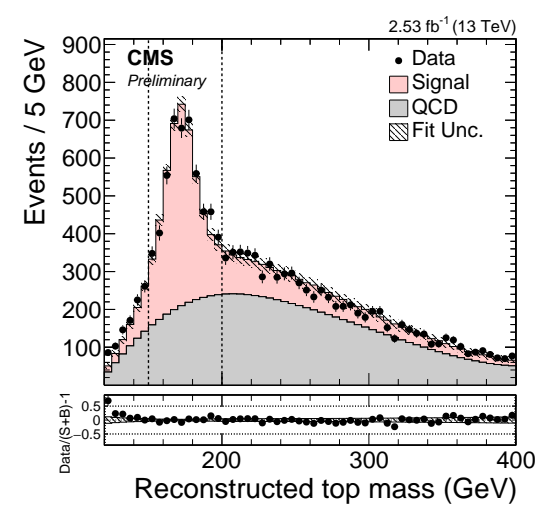

Figure 3: Reconstructed top mass distribution for data, expected signal and backgrounds in the all hadronic final state [7].

\subsection{First measurements at $\sqrt{s}=\mathbf{5 . 0 2} \mathrm{TeV}$}

In November 2015, LHC collided protons at a centre-of-mass energy of $5.02 \mathrm{TeV}$. CMS

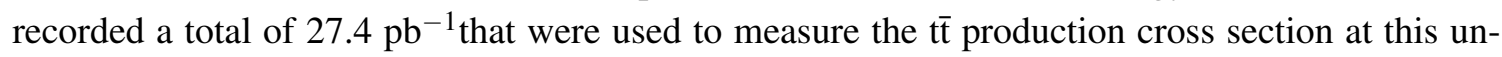
explored energy [8]. This is a very interesting region in the curve $\sigma_{t \bar{t}}(\sqrt{s})$, as $\mathrm{t} \overline{\mathrm{t}}$ events are mainly produced by large-x gluons. Therefore, the measured $t \bar{t}$ cross section at this energy can be used to constrain the gluon distribution function at large $\mathrm{x}$.

The cross section is measured using events with at least one lepton. Independent measurements in e/ $\mu+$ jets and dilepton channels are done, following similar strategies as in the previous mentioned measurements at $13 \mathrm{TeV}$ for each channel. A combination of the measurements is presented, reducing significatively the statistical uncertainty, which is dominant due to the small amount of data available at this center-of-mass energy. The result is $\sigma_{t \bar{t}}=69.5 \pm 6.1$ (stat) \pm 5.6 (syst) \pm 1.6 (lumi) pb, compatible with the SM prediction.

In Figure 4 a summary of cross sections measurements by CMS and its dependence on the centre-of-mass energy is shown.

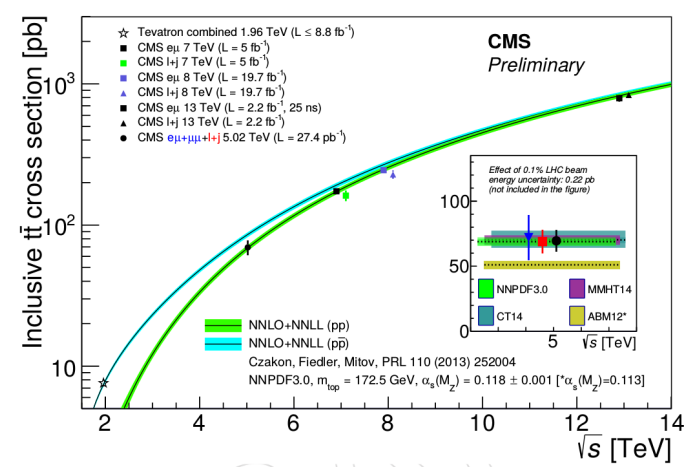

Figure 4: Summary of $t \bar{t}$ cross section measurements in CMS for various centre-of-mass energies [8].

\section{Diferential cross sections and modeling}

Recent measurements of the t⿱亠t differential production cross section have been done by CMS 
at $13 \mathrm{TeV}$ in the lepton+jets channel [9]. The results are presented at parton and particle levels and compared with several SM predictions. Also, a similar study has been done in the dilepton channel [10]. Results are compared with several SM MC predictions and available state-of-the-art QCD calculations.

Furthermore, recent studies of the double differential production cross section at $8 \mathrm{TeV}$ have been done [11]. Several pairs of observables are used to characterize the normalized cross section, showing an overall agreementwith MC predictions. This results are used to constrain PDFs.

In the mentioned studies at 8 and $13 \mathrm{TeV}$ the jet multiplicity distribution in data is found not to be well modeled by different MC generators. This has led to detailed studies of the impact of the parton shower tuning in the Pythia8 MC generator on the modeling of modeling of tî processes [12]. As a result, the newly tuned MC generators are able to improve the description of the jet multiplicity, without affecting other (more global) top quark related distributions. In Figure 5 the effect of the new tuning on the jet multiplicity on the dilepton channel is shown.

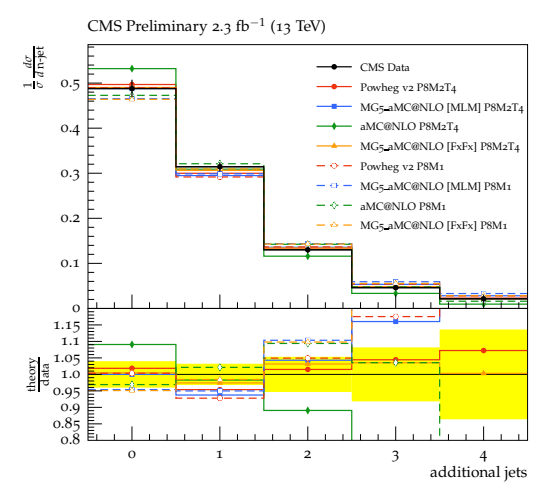

Figure 5: Distribution of jet multiplicity for events with a dilepton pair and at least two jets for different generators and the previous (P8M1) and new (P8M2T4) tune of parameters. The ratios shown are with respect to data [12].

\section{Search for the production of $t \bar{t}$ pairs in association with b quarks}

This process is a main background for the search of the ttH process, which can be used to measure the top Yukawa coupling. Thus, the accurate measurement of the $\sigma_{t t b b}$ cross section is crucial for the $\mathrm{ttH}$ measurements. Also, the ratio with the cross section of $\mathrm{t} \overline{\mathrm{t}}$ accompanied by two light jets $\left(\sigma_{t t j j}\right)$, where most of systematic uncertainties cancel out, is an excellent test for QCD.

CMS has recently presented these two measurements [13], using events with an opposite-sign dilepton pair, at least four jets and at least two $b$ jets. The signal extraction was done by fitting the b-tag discriminator for the third and fourth jets in the events. This distribution is shown in Figure 6.

The measured tt̄b $\bar{b}$ cross section is: $\sigma_{t t b b}=4.0 \pm 0.6$ (stat) \pm 1.3 (syst) pb.

\section{Search the production of four top quarks}

The four-top production process has a tiny cross section in the SM, but is very sensitive to new physics. It can also probe top Yukawa couplings. In a recently published study by CMS [14] 


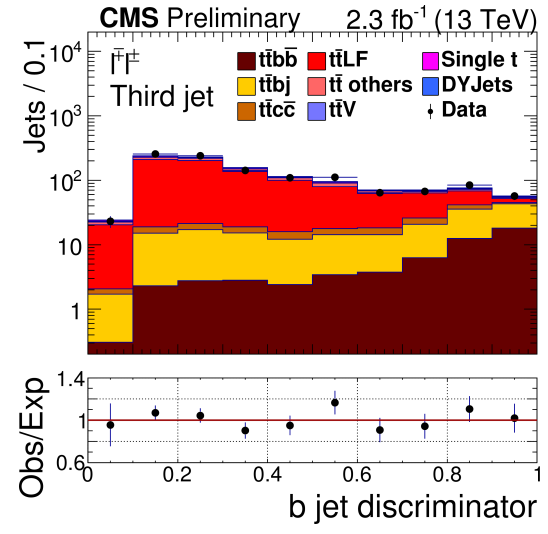

Figure 6: Distribution of the b-tag discriminator for extra jets in tțb̄ events [13].

a search of tttt production is done using events with one lepton and two opposite-sign leptons and boosted decision trees. Furthermore, a reinterpretation of a search for supersymmetry in events with two same-sign leptons [15] has helped to increase the sensitivity of the search for four-top production.

In Figure 7 the upper limits set by the search on each channel are shown. A combination of these results gives an upper limit of 4.3 times the SM cross section on the four-top production cross section.

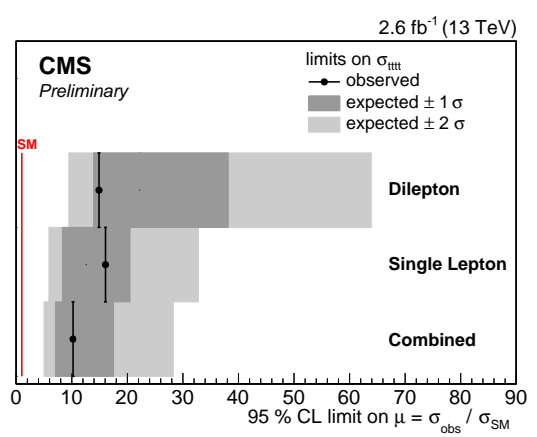

Figure 7: Upper limits on signal strength of four-top production for each explored channel and the combination [14].

\section{Summary}

Top quark measurements provide important information about the production process as described in QCD, as well as sensitivity to possible new physics. The latest measurements from CMS on tt inclusive and differential production cross sections have been presented, showing very good agreement with the state-of-the-art of SM predictions. Measurements of the $t \bar{t}$ cross section with extra jets in the event led to new tuning of parameters of the generators. New measurements of $\mathrm{t} \overline{\mathrm{t}} \mathrm{b} \overline{\mathrm{b}}$ and search for tttt $\bar{t}$ processes have been presented, obtaining results in agreement with SM expectations. 


\section{References}

[1] CMS Collaboration, "The CMS experiment at the CERN LHC", JINST, 3 (2008) S08004, doi: 10.1088/1748-0221/3/08/S08004.

[2] CMS Collaboration, "Measurement of the top quark mass using proton-proton data at $\sqrt{s}=7$ and 8 TeV”, Phys. Rev. D 93, 072004 (2016), doi: 10.1103/PhysRevD.93.072004, [arXiv:1509.04044].

[3] M. Czakon and A. Mitov, "Top++: a program for the calculation of the top-pair cross-section at hadron colliders", Comput. Phys. Commun. 185 (2014) 2930, doi: 10.1016/j.cpc.2014.06.021, [arXiv:1112.6254].

[4] CMS Collaboration, "Measurement of the top quark pair production cross section in proton-proton collisions at $\sqrt{s}=13$ TeV”, Phys. Rev. Lett. 116, 052002 (2016), doi:

10.1103/PhysRevLett.116.052002, [arXiv:1510.05302].

[5] CMS Collaboration, "Measurement of the $t \bar{t}$ production cross section using events in the $e \mu$ final state in pp collisions at $\sqrt{s}=13 \mathrm{TeV}$ ", EPJC 77 (2017) 172, 10.1103/PhysRevLett.116.052002, [arXiv:1611.04040]

[6] CMS Collaboration, "Measurement of the $t \bar{t}$ production cross section using events with one lepton and at least one jet in pp collisions at $\sqrt{s}=13$ TeV", CMS-TOP-16-006, [arXiv:1701.06228].

[7] CMS Collaboration, "Measurement of the t⿱t production cross section at $13 \mathrm{TeV}$ in the all-jets final state”, CMS Physics Analysis Summary CMS-PAS-TOP-16-013, CERN, 2017. cds.cern.ch/record/2161138/.

[8] CMS Collaboration, "Inclusive ttbar cross section at $\sqrt{s}=5.02 \mathrm{TeV}$ using final states with at least one charged lepton”, CMS Physics Analysis Summary CMS-PAS-TOP-16-023, CERN, 2017. cds.cern.ch/record/2258177/.

[9] CMS Collaboration, "Measurement of differential cross sections for top quark pair production using the lepton+jets final state in proton-proton collisions at 13 TeV”, Phys. Rev. D 95, 092001 (2017) [arXiv:1610.04191]

[10] CMS Collaboration, "Top quark pair differential cross sections at particle level in the dilepton channel at 13 TeV”, CMS-TOP-16-007, [arXiv:1708.07638].

[11] CMS Collaboration, "Measurement of double-differential cross sections for top quark pair production in pp collisions at sqrt(s) $=8 \mathrm{TeV}$ and impact on parton distribution functions", EPJC 77 (2017) 459 [arXiv:1703.01630].

[12] CMS Collaboration, "Comparisons of MC generator predictions for ttbar with data at $\operatorname{sqrt}(\mathrm{s})=8$ and 13 TeV”, CMS Physics Analysis Summary CMS-PAS-TOP-16-021, CERN, 2017. cds.cern.ch/record/2235192/.

[13] CMS Collaboration, "Measurements of ttbar cross sections in association with $b$ jets and inclusive jets and their ratio using dilepton final states in pp collisions at sqrt(s) = 13 TeV", CMS-TOP-16-010, CERN, 2017 [arXiv:1705.10141].

[14] CMS Collaboration, "Search for standard model production of four top quarks in proton-proton collisions at sqrt(s) = 13 TeV”, Phys. Lett. B 772 (2017) 336 [arXiv:1702.06164]. Additional material: cds.cern.ch/record/2205265/files/.

[15] CMS Collaboration, "Search for physics beyond the standard model in events with two leptons of same sign, missing transverse momentum, and jets in proton-proton collisions at sqrt(s)=13 TeV", CMS-SUS-16-035, CERN, 2017 [arXiv:1704.07323]. 